\title{
NOTES ON COMPLEX HYPERBOLIC FUNCTIONS
}

\author{
S. Owa, K. Toyoda, S. Ebata, M. Nakamura, T. Yamamoto, R. Yamato
}

Abstract. Noting the derivatives for functions $\sinh z$ and $\cosh z$, we assume the fractional derivatives for $\sinh z$ and $\cosh z$. Applying the fractional calculus (fractional integrals and fractional derivatives), we consider generalized expansions for functions $\sinh z$ and $\cosh z$. Also, the generalized expansion for $f(z)=e^{z}$ is considered.

\section{Mathematics Subject Classification: 30C45.}

Keywords: Analytic function, Taylor expansion, fractional calculus, complex hyperbolic function.

\section{INTRODUCTION}

Let $\mathcal{A}(\alpha)$ be the class of functions $f(z)$ of the form

$$
\begin{gathered}
f(z)=a_{0} z^{\alpha}+a_{1} z^{\alpha+1}+a_{2} z^{\alpha+2}+\cdots \\
=\sum_{n=0}^{\infty} a_{n} z^{\alpha+n}
\end{gathered}
$$

for $0 \leqq \alpha<1$ which are analytic in the open unit disk $\mathbb{U}=\{z \in \mathbb{C}:|z|<1\}$. If $\alpha=0$ in (1.1), then $f(z) \in \mathcal{A}(0)$ becomes

$$
f(z)=a_{0}+a_{1} z+a_{2} z^{2}+\cdots=\sum_{n=0}^{\infty} a_{n} z^{n},
$$

and that

$$
f(z)=f(0)+\frac{f^{\prime}(0)}{1 !} z+\frac{f^{\prime \prime}(0)}{2 !} z^{2}+\cdots=\sum_{n=0}^{\infty} \frac{f^{(n)}(0)}{n !} z^{n} .
$$

This is the orginary Taylor expansion for $f(z) \in \mathcal{A}(0)$. Therefore, for $f(z) \in \mathcal{A}(\alpha)$, we have to consider the generalized Taylor expansion of $f(z)$. 
To consider this problem, we have to use the fractional calculus (fractional integrals and fractional derivatives) defined by Owa [1], Owa and Srivastava [2], and Srivastava and Owa [3].

Definition 1.1 The fractional integral of order $\alpha$ is defined, for an analytic function $f(z)$ in $\mathbb{U}$, by

$$
D_{z}^{-\alpha} f(z)=\frac{1}{\Gamma(\alpha)} \int_{0}^{z} \frac{f(t)}{(z-t)^{1-\alpha}} d t \quad(\alpha>0)
$$

where the multiplicity of $(z-t)^{\alpha-1}$ is removed by requiring $\log (z-t)$ to be real when $z-t>0$.

Definition 1.2 The fractional derivative of order $\alpha$ is defined, for an analytic function $f(z)$ in $\mathbb{U}$, by

$$
D_{z}^{\alpha} f(z)=\frac{d}{d z}\left(D_{z}^{\alpha-1} f(z)\right)=\frac{1}{\Gamma(1-\alpha)} \frac{d}{d z}\left(\int_{0}^{z} \frac{f(t)}{(z-t)^{\alpha}} d t\right)
$$

where $0 \leqq \alpha<1$ and the multiplicity of $(z-t)^{-\alpha}$ is removed as Definition 1.1 above.

Definition 1.3 Under the hypotheses of Definition 1.2, the fractional derivative of order $n+\alpha$ is defined by

$$
D_{z}^{n+\alpha} f(z)=\frac{d^{n}}{d z^{n}}\left(D_{z}^{\alpha} f(z)\right),
$$

where $0 \leqq \alpha<1$ and $n \in \mathbb{N}_{0}=\{0,1,2, \cdots\}$.

By means of Definition 1.2, we have that

$$
\begin{gathered}
D_{z}^{\alpha} z^{\alpha+n}=\frac{d}{d z}\left(D_{z}^{\alpha-1} z^{\alpha+n}\right) \\
=\frac{d}{d z}\left\{\frac{1}{\Gamma(1-\alpha)} \int_{0}^{z} \frac{t^{\alpha+n}}{(z-t)^{\alpha}} d t\right\} \\
=\frac{1}{\Gamma(1-\alpha)} \frac{d}{d z}\left\{z^{n+1} \int_{0}^{1} \frac{(1-\zeta)^{\alpha+n}}{\zeta^{\alpha}} d \zeta\right\} \quad(z-t=z \zeta) \\
=\frac{1}{\Gamma(1-\alpha)} \frac{d}{d z}\left(z^{n+1} B(1-\alpha, \alpha+n+1)\right)=\frac{\Gamma(\alpha+n+1)}{\Gamma(n+1)} z^{n},
\end{gathered}
$$

where $B(x, y)$ is the beta function. Therefore, we know, for $f(z) \in \mathcal{A}(\alpha)$, that

$$
D_{z}^{\alpha} f(z)=D_{z}^{\alpha}\left(\sum_{n=0}^{\infty} a_{n} z^{\alpha+n}\right)=\sum_{n=0}^{\infty} \frac{\Gamma(\alpha+n+1)}{\Gamma(n+1)} a_{n} z^{n} .
$$


With the above, we know that

$$
a_{0}=\frac{D_{z}^{\alpha} f(0)}{\Gamma(\alpha+1)}
$$

Using

$$
D_{z}^{\alpha+1} f(0)=\Gamma(\alpha+2) a_{1}
$$

we can write that

$$
a_{1}=\frac{D_{z}^{\alpha+1} f(0)}{\Gamma(\alpha+2)}
$$

In general, we see that

$$
a_{n}=\frac{D_{z}^{\alpha+n} f(0)}{\Gamma(\alpha+n+1)}
$$

for $0 \leqq \alpha<1$ and $n \in \mathbb{N}_{0}$. Consequently, we have that

$$
f(z)=\sum_{n=0}^{\infty} \frac{D_{z}^{\alpha+n} f(0)}{\Gamma(\alpha+n+1)} z^{\alpha+n}
$$

for $f(z) \in \mathcal{A}(\alpha)$ with $z \neq 0$. Therefore, we use this expansion (1.13) for $f(z) \in \mathcal{A}(\alpha)$.

\section{EXPANSIONS FOR TRIGONOMETRIC FUNCTIONS}

We discuss the expansions for complex hyperbolic funtions $\sinh z$ and $\cosh z$ for $z \in \mathbb{U}$. Noting that

$$
\sinh z=\frac{e^{z}-e^{-z}}{2}
$$

and

$$
\cosh z=\frac{e^{z}+e^{-z}}{2}
$$

we obtain that

$$
\sin i z=\frac{e^{-z}-e^{z}}{2 i}=i \sinh z,
$$


that is, that

$$
\sinh z=\frac{1}{i} \sin (i z)
$$

Therefore, if $f(z)=\sinh z$, then

$$
\begin{gathered}
f^{\prime}(z)=\cos (i z)=\sin \left(i z+\frac{\pi}{2}\right), \\
f^{\prime \prime}(z)=i \cos \left(i z+\frac{\pi}{2}\right)=i \sin (i z+\pi),
\end{gathered}
$$

and that

$$
f^{(n)}(z)=i^{n-1} \sin \left(i z+\frac{n}{2} \pi\right) \quad\left(n \in \mathbb{N}_{0}\right) .
$$

With the above, we may assume that

$$
D_{z}^{\alpha} f(z)=i^{\alpha-1} \sin \left(i z+\frac{\alpha}{2} \pi\right) \quad(0 \leqq \alpha<1)
$$

and

$$
D_{z}^{\alpha+n} f(z)=i^{\alpha+n-1} \sin \left(i z+\frac{\alpha+n}{2} \pi\right)
$$

for $0 \leqq \alpha<1$ and $n \in \mathbb{N}_{0}$.

Remark 2.1 Applying the formula (2.8), we see that

$$
f^{(n)}(z)=D_{z}^{n-\alpha}\left(D_{z}^{\alpha} f(z)\right)=D_{z}^{n-\alpha}\left(i^{\alpha-1} \sin \left(i z+\frac{\alpha}{2} \pi\right)\right)=i^{n-1} \sin \left(i z+\frac{n}{2} \pi\right)
$$

for $0 \leqq \alpha<1$ and $n \in \mathbb{N}_{0}$.

Now, we derive

Theorem 2.1 If the equation (2.8) is satisfied for $f(z)=\sinh z$, then

$$
\sinh z=\sum_{n=0}^{\infty} \frac{i^{\alpha+n-1} \sin \left(\frac{\alpha+n}{2} \pi\right)}{\Gamma(\alpha+n+1)} z^{\alpha+n} \quad(z \in \mathbb{U}-\{0\})
$$

where $0 \leqq \alpha<1$.

Proof By means of (2.8) and (2.9), we know that

$$
D_{z}^{\alpha} f(0)=i^{\alpha-1} \sin \left(\frac{\alpha}{2} \pi\right) \quad(0 \leqq \alpha<1)
$$


and

$$
D_{z}^{\alpha+n} f(0)=i^{\alpha+n-1} \sin \left(\frac{\alpha+n}{2} \pi\right) \quad\left(0 \leqq \alpha<1, n \in \mathbb{N}_{0}\right)
$$

eachother. Therefore, using (1.13), we complete the proof of the theorem.

Making $\alpha=\frac{1}{2}$ in Theorem 2.1, we have

Corollary 2.1 If the equation (2.8) is satisfied for $f(z)=\sinh z$ with $\alpha=\frac{1}{2}$, then

$$
\begin{gathered}
\sinh z=\sum_{n=0}^{\infty} \frac{i^{n-\frac{1}{2}} \sin \left(\frac{2 n+1}{4} \pi\right)}{\Gamma\left(n+\frac{3}{2}\right)} z^{n+\frac{1}{2}} \\
=-\frac{\sqrt{2} \sqrt{z}}{\sqrt{\pi}}\left(1+\frac{2 i}{3} z-\frac{2^{2}}{3 \cdot 5} z^{2}-\frac{2^{3} i}{3 \cdot 5 \cdot 7} z^{3}\right. \\
\left.+\frac{2^{4}}{3 \cdot 5 \cdot 7 \cdot 9} z^{4}+\frac{2^{5} i}{3 \cdot 5 \cdot 7 \cdot 9 \cdot 11} z^{5}-\cdots\right)
\end{gathered}
$$

for $z \in \mathbb{U}-\{0\}$.

Further, we consider the function $\cosh z$ for $\mathbb{U}$. Note that

$$
f(z)=\cosh z=\frac{e^{z}+e^{-z}}{2}=\cos (i z) .
$$

This gives us that

$$
\begin{gathered}
f^{\prime}(z)=-i \sin (i z)=i \cos \left(i z+\frac{\pi}{2}\right), \\
f^{\prime \prime}(z)=-i^{2} \sin \left(i z+\frac{\pi}{2}\right)=i^{2} \cos (i z+\pi),
\end{gathered}
$$

and that

$$
f^{n}(z)=i^{n} \cos \left(i z+\frac{n}{2} \pi\right) \quad\left(n \in \mathbb{N}_{0}\right) .
$$

From the above, we can assume that

$$
D_{z}^{\alpha} f(z)=i^{\alpha} \cos \left(i z+\frac{\alpha}{2} \pi\right) \quad(0 \leqq \alpha<1)
$$

and

$$
D_{z}^{\alpha+n} f(z)=i^{\alpha+n} \cos \left(i z+\frac{\alpha+n}{2} \pi\right)
$$


for $0 \leqq \alpha<1$ and $n \in \mathbb{N}_{0}$.

Remark 2.2 Using the formula (2.19), we have that

$$
f^{(n)}(z)=D_{z}^{n-\alpha}\left(D_{z}^{\alpha} f(z)\right)=D_{z}^{n-\alpha}\left(i^{\alpha} \cos \left(i z+\frac{\alpha}{2} \pi\right)\right)=i^{n} \cos \left(i z+\frac{n}{2} \pi\right)
$$

for $0 \leqq \alpha<1$ and $n \in \mathbb{N}_{0}$.

Now, we have

Theorem 2.2 If the equation (2.19) is satisfied for $f(z)=\cosh z$, then

$$
\cosh z=\sum_{n=0}^{\infty} \frac{i^{\alpha+n} \cos \left(\frac{\alpha+n}{2} \pi\right)}{\Gamma(\alpha+n+1)} z^{\alpha+n} \quad(z \in \mathbb{U}-\{0\})
$$

with $0 \leqq \alpha<1$.

Proof Note that

$$
D_{z}^{\alpha} f(0)=i^{\alpha} \cos \left(\frac{\alpha}{2} \pi\right) \quad(0 \leqq \alpha<1)
$$

and

$$
\left.D_{z}^{\alpha+n} f(0)=i^{\alpha+n} \cos \left(\frac{\alpha+n}{2} \pi\right) \quad(0 \leqq \alpha<1), n \in \mathbb{U}-\{0\}\right) .
$$

Therefore, the formula (1.13) implies the equality (2.22).

Putting $\alpha=\frac{1}{2}$ in Theorem 2.2, we have

Corollary 2.2 If the equation (2.19) is satisfied for $\alpha=\frac{1}{2}$, then

$$
\begin{gathered}
\cosh z=\sum_{n=0}^{\infty} \frac{i^{n+\frac{1}{2}} \cos \left(\frac{2 n+1}{4} \pi\right)}{\Gamma\left(n+\frac{3}{2}\right)} z^{n+\frac{1}{2}} \\
=\frac{\sqrt{2} \sqrt{z} i}{\sqrt{\pi}}\left(1-\frac{2 i}{3} z+\frac{2^{2}}{3 \cdot 5} z^{2}-\frac{2^{3} i}{3 \cdot 5 \cdot 7} z^{3}\right. \\
\left.+\frac{2^{4}}{3 \cdot 5 \cdot 7 \cdot 9} z^{4}-\frac{2^{5}}{3 \cdot 5 \cdot 7 \cdot 9 \cdot 11} z^{5}-\cdots\right)
\end{gathered}
$$

for $z \in \mathbb{U}-\{0\}$.

Finally, we derive

Theorem 2.3 If the equations (2.8) for $\sinh z$ and (2.19) for $\cosh z$ are satisfied, then

$$
e^{z}=\sum_{n=0}^{\infty} \frac{i^{\alpha+n} \cos \left(\frac{\alpha+n}{2} \pi\right)-i \sin \left(\frac{\alpha+n}{2} \pi\right)}{\Gamma(\alpha+n+1)} z^{\alpha+n} \quad(z \in \mathbb{N}-\{0\})
$$


with $0 \leqq \alpha<1$ for $z \in \mathbb{U}-\{0\}$.

Proof Since

$$
e^{z}=\cosh z-\sinh z
$$

using Theorem 2.1 and Theorem 2.2, we see (2.26).

If we take $\alpha=\frac{1}{2}$ in Theorem 2.3, we obtain

Corollary 2.3 If the equations (2.8) and (2.19) are satisfied for $\alpha=\frac{1}{2}$, then

$$
\begin{aligned}
e^{z}= & \frac{\sqrt{2} \sqrt{z} \sqrt{i}}{\sqrt{\pi}}\left\{(1-i)-\frac{2}{3}(1+i) z-\frac{2^{2}}{3 \cdot 5}(1-i) z^{2}+\frac{2^{3}}{3 \cdot 5 \cdot 7}(1+i) z^{3}\right. \\
& \left.+\frac{2^{4}}{3 \cdot 5 \cdot 7 \cdot 9}(1-i) z^{4}-\frac{2^{5}}{3 \cdot 5 \cdot 7 \cdot 9 \cdot 11}(1+i) z^{5}+\cdots\right)
\end{aligned}
$$

for $z \in \mathbb{U}-\{0\}$.

\section{REFERENCES}

[1] S. Owa, On the distortion theorem, I, Kyungpook Math. J. 18(1978), 53 - 59.

[2] S. Owa and H. M. Srivastava, Univalent and starlike generalized hypergeometric functions, Canad. J. Math. 39(1987), 1057 - 1077.

[3] H. M. Srivastava and S. Owa, Some characterization and distortion theorems involving fractional calculus, generalized hypergeometric functions, Hadamard products, linear operators, and certain subclasses of analytic functions, Nagoya Math. J. 106(1987), $1-28$.

\section{Shigeyoshi Owa}

Department of Mathematics

Faculty of Education

Yamato University

Suita, Osaka 564-0082, Japan

e-mail: owa.shigeyoshi@yamato-u.ac.jp

Koichi Toyoda

Department of Mathematics

Faculty of Education

Yamato University

Suita, Osaka 564-0082, Japan 
S. Owa, K. Toyoda, S. Ebata, M. Nakamura, T. Yamamoto, R. Yamato - . .

Shunya Ebata

Department of Mathematics

Faculty of Education

Yamato University

Suita, Osaka 564-0082, Japan

Masatomo Nakamura

Department of Mathematics

Faculty of Education

Yamato University

Suita, Osaka 564-0082, Japan

Taketsugu Yamamoto

Department of Mathematics

Faculty of Education

Yamato University

Suita, Osaka 564-0082, Japan

Ryota Yamato

Department of Mathematics

Faculty of Education

Yamato University

Suita, Osaka 564-0082, Japan 